\title{
Compressed Sensing Reconstruction of Convolved Sparse Signals
}

\author{
Grigorios Tsagkatakis \\ Panagiotis Tsakalides \\ ICS-FORTH, Greece
}

\author{
Arnaud Woiselle \\ Marc Bousquet \\ Sagem Défense Sécurité, France
}

\author{
George Tzagkarakis \\ Jean-Luc Starck \\ CEA, Service d'Astrophysique, France
}

\begin{abstract}
This paper addresses the problem of efficient sampling and reconstruction of sparse spike signals, which have been convolved with low-pass filters. A modified compressed sensing (CS) framework is proposed, termed dictionary-based deconvolution CS (DDCS) to achieve this goal. DDCS builds on the assumption that a low-pass filter can be represented sparsely in a dictionary of blurring atoms. Identification of both the sparse spike signal and the sparsely parameterized blurring function is performed by an alternating scheme that minimizes each variable independently, while keeping the other constant. Simulation results reveal that the proposed DDSS scheme achieves an improved reconstruction performance when compared to traditional CS recovery.
\end{abstract}

\section{INTRODUCTION}

According to the well-established theories of Shannon and Nyquist, the exact recovery of a discrete signal requires a sampling rate twice the signal's bandwidth. Moreover, the sampling scheme characteristics can have dramatic consequences on the quality of the recorded signals, the hardware necessary to achieve the required quality and therefore the cost, time and effort that accompany the process. As a result, designing efficient sampling schemes is a critical part of numerous applications. In this work, we focus on a specific case of signals, which can be modeled as time-series of shifted and scaled pulses.

This class of signals encompasses a large number of signals, ranging from neuronal activity [1] and ultrasound imaging [2], to radar [3], UWB communications [4] and physiological signals [5]. As an illustrative example, consider the case of range imaging data obtained by a Time-of-Flight (ToF) camera. Such systems function by emitting a laser pulse which propagates through the atmosphere, is reflected by an object and is subsequently recorded by an imaging sensor. Depth information encoding is achieved by opening the electronic gate of the camera only during a specific time interval, which corresponds to pulses from a specific depth range.

A major limitation of this type of sampling scheme is that, in order to obtain a full range sweep, that is to obtain distance measurements for objects at $n$ discrete depth levels, $n$ pulses have to be recorded. This process results in a large number of unnecessary measurements that may contain no valid information at all, since we expect that only a single, or very few, measurements to actually record reflected pulses.

To overcome this apparent waste of resources, a novel theory in signal sampling and reconstruction, termed Compressed
Sensing (CS), has been recently proposed by Donoho [6] and by Candés et al. [7]. The main concept of CS is that a signal can be recovered from a small number of random measurements, far below the Nyquist-Shannon limit, provided that the signal is sparse and an appropriate sampling scheme is employed.

By modeling the pulses as a series of spike (delta) functions, CS theory suggests that recovering the sparse spike signals is possible if enough random measurements of the form $\mathbf{y}=\boldsymbol{\Phi} \mathbf{x} \in \mathbb{R}^{M}$ with $M \ll N$ are taken, and the $M \times N$ sampling matrix $\Phi$ adheres to the RIP property [8]. Recovery of the sparse signal can be achieved by solving the following $\ell_{1}$ minimization problem:

$$
\min _{\mathbf{x}}\|\mathbf{x}\|_{1} \text { subject to }\|\mathbf{y}-\mathbf{\Phi} \mathbf{x}\|_{2}^{2}<\epsilon
$$

where $\|x\|_{1}=\sum_{i}\left|x_{i}\right|$ is the $\ell_{1}$ norm, introduced as the convex surrogate of the non-zero counting $\ell_{0}$ pseudo-norm, which make the optimization tractable. Additionally, $\epsilon$ accounts for noise and approximation errors. This problem is known as the basis pursuit denoising and has recently shown to produce accurate approximation of sparse signals [9]. In addition, for signals that are strictly non-negative, such a constraint can be introduced in the optimization [10].

An extension of this scheme considers the case where the signal in question is not sparse by itself, but can be represented sparsely in a dictionary of atoms, such that $\mathbf{x}=\mathbf{D z}$ and $\|\mathbf{z}\|_{0} \leqslant K$ [7], [11]. In this case, the problem can be formulated according to the synthesis framework and the minimization is expressed as:

$$
\min _{\mathbf{z}}\|\mathbf{z}\|_{1} \text { subject to }\|\mathbf{y}-\mathbf{\Phi D \mathbf { z }}\|_{2}^{2}<\epsilon .
$$

While the above formulations of traditional CS and the dictionary extension cover a large number of signal models, considering the specific framework of sampling blurred spike trains, a main drawback of the above formulation is that it does not take into account the convolution of the spike signals with low-pass filters, which encodes the signal propagation through a medium, along with the sampling process.

An important byproduct of the convolution is that the filtering process will distribute the sparse signal's energy to a larger support, resulting in significantly less sparse signals, necessitating higher CS sampling rates. The process of signal blurring and random sampling is visually depicted in Fig. 1. 


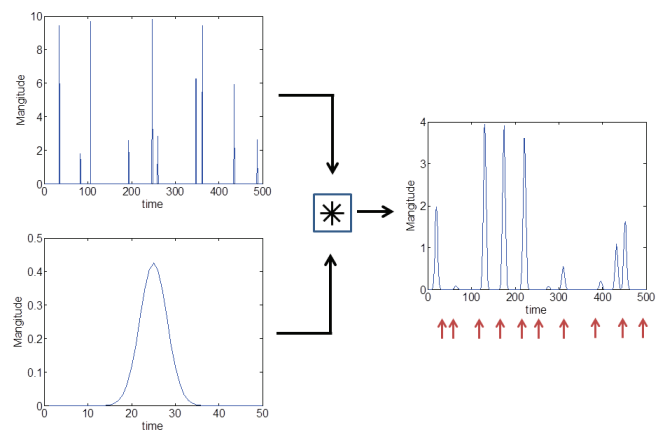

Fig. 1. Visual illustration of the blurring process. The sparse spike train signal (top left) is convolved with the low-pass blurring filter (lower left), which process the blurred signal (right). One can easily observe the dramatic increase in the support of the initial sparse signal. The arrows under the horizontal (time) axes indicate the random CS-based sampling of the signal.

In this work, we propose a CS reconstruction method, which, in addition to the sparsity of the spike signal, also assumes the existence of a dictionary that is able to represent sparsely the low-pass filtering signal. Formally, let $\mathrm{s}$ be a sparse signal encoding the locations and the amplitudes of the spikes in the signal to be recovered. Furthermore, the sparse signal undergoes a convolution with a low-pass filter $\mathbf{h}$ prior to its sampling by a measurement matrix $\boldsymbol{\Phi}$, resulting in a signal $\mathbf{y}=\boldsymbol{\Phi}(\mathbf{s} * \mathbf{h})$. The objective of our proposed DDCS is to identify both $\mathbf{s}$ and $\mathbf{h}$ by exploiting the knowledge that $\mathbf{s}$ is sparse and that $\mathbf{h}$ has a sparse representation over a dictionary of blurring kernels $\mathbf{B}$.

The rest of the paper is organized as follows: Section II presents an overview of work along similar directions with the one considered here. Section III provides a description of the various signal models considered by our proposed scheme, while the proposed scheme is presented in Section IV. Experimental results are given in Section V and the paper concludes in Section VI.

\section{PREVIOUS WORK}

The problem of blind and semi-blind deconvolution of sparse signals has been explored from multiple aspects such as Bayesian approaches [12] and sparsity seeking approaches [13]. However, these methods assume that the fully sampled data is available. In a recent work [14], the recovery of the sparse signals and the filtering function from compressed measurements was carried out by an iterative scheme, where at each iteration an alternating minimization between the spike stream identification and the impulse response estimation takes place. However, unlike our work, the estimation of both signals is obtained by a least squares minimization, whereas we employ an $\ell_{1}$ regularization in order to encode sparsity assumptions and achieve high quality reconstruction. Furthermore, we assume that a parametric model of the convolution function is known and the specific realization is of interest. In [4], the CS-based sampling and reconstruction of convolved signals was also considered. In this case however, the specific form of the impulse response was assumed known, unlike this work where only a parametric model is assumed.
Recently, the framework of finite rate of innovation sampling was introduced, which considers the sampling and reconstruction of sparse signals by analog systems [15]. Our work differs in that we do not consider analog signal but discritized depth signals.

\section{Signal Models}

\section{A. Pulse train model}

In the following, we consider signals which are expressed as linear combinations of shifted and scaled delta functions,

$$
\mathbf{s}(t)=\sum_{j=1}^{K} a_{j} \delta\left(t-t_{j}\right),\left\{a_{j}\right\}_{j=1}^{K} \geqslant 0 .
$$

In essence, the signals to be recovered correspond to a sparse collection of scaled delta functions at specific time instances. Due to its construction process, the signal $\mathbf{s}$ and therefore the coefficients $a_{j}$ are strictly non-negative, a property that we exploit during the reconstruction process. Recovery of this type of signal according to the CS framework can be accomplished by collecting

$$
M=\mathcal{O}(K \log (N / K))
$$

random measurements. However, the signal is question further undergoes convolution, which will reduce the effective sparsity and thus will require a higher number of measurements for reconstruction. In this work, we address this issue by considering a blurring kernel that is not itself sparse, but can be sparsely represented in an appropriate dictionary.

\section{B. Dictionary model}

In our proposed approach, we assume that a dictionary of $L$ prototypical blurring functions encoded in $\mathbf{B}=\{\mathbf{b}\}_{i=1}^{L} \in$ $\mathbb{R}^{W \times L}$ is available. This dictionary is constructed based on the assumption that an accurate approximation of the blurring function can be achieved by selecting a small number of elements from the dictionary. The specific blurring kernel can thus be expressed according to $\mathbf{h}=\mathbf{B k}$, where $\mathbf{k}$ is a $K$-sparse signal.

In this work we consider a dictionary consisting of a collection of zero-mean Gaussian functions with different variances given by $\mathbf{b}_{i}=\frac{1}{\sqrt{2 \pi \sigma_{i}^{2}}} \exp ^{\frac{-x}{2 \sigma_{i}^{2}}}$. Furthermore, the bandwidth of the kernels are truncated to $B$ bins. The vector produced by linear combinations of dictionary elements corresponds to the realization of a Gaussian blurring kernel $\mathbf{h}=\sum_{j=1}^{L} k_{j} \mathbf{b}_{j}=$ $\sum_{j=1}^{L} \frac{1}{\sqrt{2 \pi\left(k_{j} \sigma_{j}\right)^{2}}} \exp ^{\frac{-x}{2\left(k_{j} \sigma_{j}\right)^{2}}}$, where $\left\{k_{j}\right\}_{j=1}^{L}$ are the sparse coefficients associated with the blurring kernel.

\section{Sampling matrix model}

Generating the set of random measurements of a signal according to the CS specification presents various obstacles, since the physical realization of many sampling matrices, which satisfy the necessary requirements, is quite challenging. Examples of such matrices include matrices where the elements are drawn from Gaussian and sub-Gaussian distributions [16]. However, various schemes for designing sampling 
matrices, which are supported by real hardware have been proposed recently.

In this work, the random measurements are generated by employing binary sparse matrices with a bounded number of non-zero elements per column [17]. More specifically, the sampling matrix is constructed such that each element is drawn according to:

$$
\phi_{i j}= \begin{cases}1 / \sqrt{d} & \text { with probability } \frac{d n}{k} \\ 0 & \text { otherwise }\end{cases}
$$

The performance characteristics of this type of sampling matrices were explored recently in [18], where it was shown that such random sparse binary sampling matrices satisfy the RIP, a necessary property for the recovery of signals in the framework of CS. An important advantage of these sampling matrices is that they approximate the performance of their dense counterparts, but at a significantly reduced computational and memory cost.

\section{Proposed Signal Recovery}

By considering the periodic extensions of the acquired signals, the convolution can be written as a multiplication with a circular matrix. In our specific setup, the property is expressed as:

$$
\mathbf{y}=\boldsymbol{\Phi}(\mathbf{s} * \mathbf{B k})=\mathbf{\Phi} \mathbb{C}\{\mathbf{s}\} \mathbf{B k}=\mathbf{\Phi} \mathbf{s} \mathbb{C}\{\mathbf{B k}\}=\mathbf{\Phi} \mathbf{s} \mathbb{C}\{\mathbf{h}\},
$$

where $\mathbb{C}\{\cdot\}$ is an operator that produces a Toeplitz matrix by performing the circular convolution of the signal with an appropriately sized identity matrix. The ability to express the convolution in such a way stems from the commutativity property of the convolution [19].

In this work, we further assume that the low-pass filter can be represented sparsely in a dictionary, that is, $\mathbf{h}=\mathbf{B k}$, with $\mathbf{k}$ being sparse and that the pulse train signal consist of non-negative elements. According to these assumptions, the formulation of the recovery problem is given by:

$$
\begin{aligned}
\min _{\mathbf{s}, \mathbf{k}} & \|\mathbf{s}\|_{1}+\|\mathbf{k}\|_{1} \\
\text { subject to } & \|\mathbf{y}-\mathbf{\Phi}(\mathbf{s} * \mathbf{B k})\|_{2}^{2}<\epsilon \\
& \mathbf{s} \geqslant \mathbf{0} .
\end{aligned}
$$

One can observe that the objective is the sum of two convex functions, while the constraint encodes the pulse train generation process, that is, the sampling of the two convolved signals. Furthermore, the requirement for a non-negative pulse train signal is also encoded in the constraint. Solving the above minimization problem is a challenging task due to the coupling of the variables in the objective. However, owning to the commutative property of the convolution, we employ an alternating minimization that capitalizes on the separability of the variables when they are considered individually.

\section{A. Alternating minimization}

In order to solve efficiently the problem expressed by Eq. (7), we employ an alternating minimization scheme, where during each iteration we minimize alternatively over one variable of the problem in Eq. (7) and keeping the rest of the variables fixed. This alternating optimization approach has been utilized extensively for deconvolution, where constraints on both the estimated signal and the blurring kernel are necessary [20]. More specifically, minimizing with respect to the pulse train signal $\mathbf{s}^{t}$, the optimization is given by

$$
\begin{aligned}
\min & \left\|\mathbf{s}^{t}\right\|_{1} \\
\text { subject to } & \left\|\mathbf{y}-\mathbf{\Phi} \mathbf{G} \mathbf{s}^{t}\right\|_{2}^{2}<\epsilon_{1},
\end{aligned}
$$

where the matrix $\mathbf{G}=\mathbb{C}\left\{\mathbf{h}^{t-1}\right\}=\mathbb{C}\left\{\mathbf{B k}^{t-1}\right\}$ contains shifted versions of the estimated blurring function parameterized by $\mathbf{k}^{t-1}$. Similarly, minimizing Eq. (7) with respect to $\mathbf{k}^{t}$, the optimization is given by:

$$
\begin{aligned}
\min & \left\|\mathbf{k}^{t}\right\|_{1} \\
\text { subject to } & \left\|\mathbf{y}-\mathbf{\Phi} \mathbf{Q} \mathbf{k}^{t}\right\|_{2}^{2}<\epsilon_{2},
\end{aligned}
$$

where $\mathbf{Q}=\mathbb{C}\left\{\mathbf{s}^{t-1}\right\} \mathbf{B}$ encodes multiple blurred versions of the estimated pulse train signal, each one with a different blurring kernel. The process continues to iterate until a maximum iterations number is reached or a threshold on the error is surpassed.

Due to the coupling of the variables in the objective function in Eq. (7), global optimality is not guaranteed. Fortunately, high quality approximations can be found if the optimal solution to each subproblem is found [21]. One can observe that the minimization problems in Eqs. (8) and (9) are instances of $\ell_{1}$-regularized minimization and more specifically the dictionary based formulation where the signals in question can be represented sparsely in the corresponding dictionaries, namely, $\mathbf{G}$ and $\mathbf{Q}$.

Due to the specific construction process of the dictionary and the sampling matrix, we expect that the coherence between the dictionary and the sensing matrix to be low. The low coherence, which suggest low sampling rates, is expected since the non-zero elements of the sampling matrix are spread while the dictionary elements are highly localized. In the extreme case where the blur kernel corresponds to a constant value (infinite bandwidth), the maximum of the inner product between a column of the sensing matrix and the dictionary element will be 0.5 .

\section{EXPERIMENTAL RESULTS}

In this section, we evaluate the performance of our proposed method in terms of the achieved reconstruction error against the standard CS reconstruction on synthetically generated data. We consider the reconstruction of sparse pulse train signals $\mathbf{s} \in \mathbb{R}^{500}$, which have undergone convolution with blurring kernels $\mathbf{h} \in \mathbb{R}^{50}$ and sampled with a binary sampling matrix $\boldsymbol{\Phi}$ with degree equal to 0.5 . The blurring dictionary is constructed by taking 100 Gaussians function with variance ranging from 0.1 to 5 , resulting in a $50 \times 100$ blurring dictionary. The reconstruction performance of the sparse signals under various sparsity, blurring kernel bandwidth and sampling ratio parameters is examined. We limited the number of iterations of the 
DDCS to 10 , since we did not observe significant change in performance with larger number of iterations.

Figs. 2 and 3 present the reconstruction performance of the sparse pulse train with blurring kernel variances equal to $\sigma^{2}=1.9$ and 3.1. Examining the first plot, we observe that under all sampling rates, the proposed DDCS offers better reconstruction quality compared to traditional CS. Furthermore, the reconstruction error reduces more smoothly for the case of DDCS compared to traditional CS at medium sampling rates. As it was expected, the performance is better for pulse train signals with increased sparsity. Considering the more challenging case shown in Fig. 3, we again observe the superiority of the reconstruction of DDCS versus traditional CS. The case of reconstructing signals that have been additionally infected by Gaussian noise is presented in Fig. 4. Last, the quality of the blur kernel estimation is shown in Fig. 5.

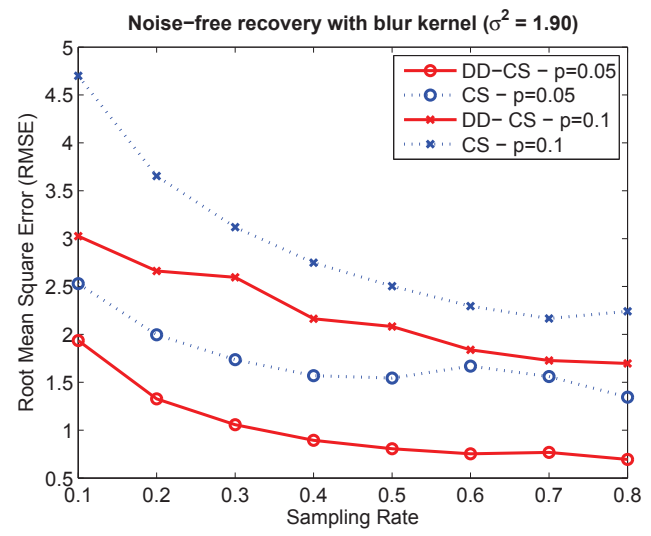

Fig. 2. Reconstruction error for standard CS (blue) and proposed scheme (red) for signals with 5\% ('x') and 10\% ('x') sparsity that have been convolved with Gaussian filters of $\sigma^{2}=1.9$. We observe that the proposed scheme achieves better reconstruction in both cases, especially for signals of lower sparsity and more strongly filtered.

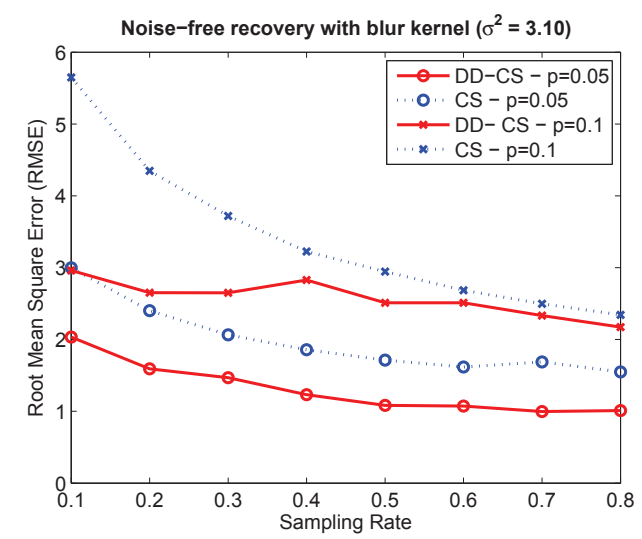

Fig. 3. Reconstruction error for standard CS (blue) and proposed scheme (red) for signals with 5\% ('x') and 10\% ('o') sparsity that have been convolved with Gaussian filters of $\sigma^{2}=3.1$. Compared to Fig. 2, we observe that the proposed DDCS is more robust to more heavily filtered signals compared to standard CS

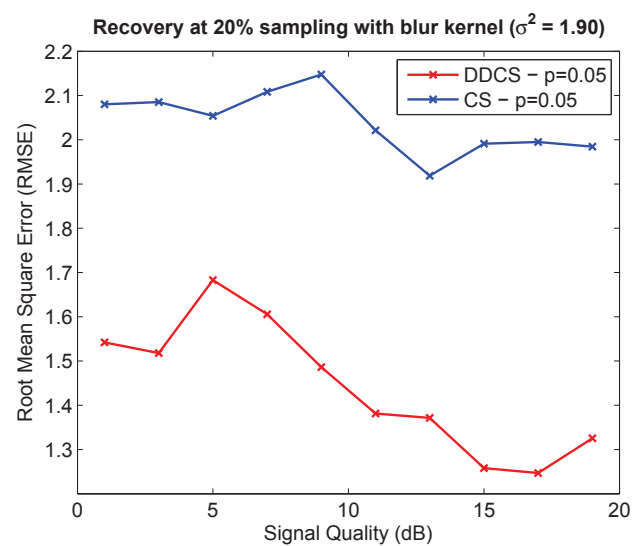

Fig. 4. Reconstruction error at $20 \%$ sampling rate in noisy conditions for standard CS (blue) and proposed scheme (red) for signals with $5 \%$ sparsity that have been convolved with Gaussian filters of $\sigma^{2}=1.9$. The horizontal axis indicates different levels of signal quality, expressed in $\mathrm{dB}$.

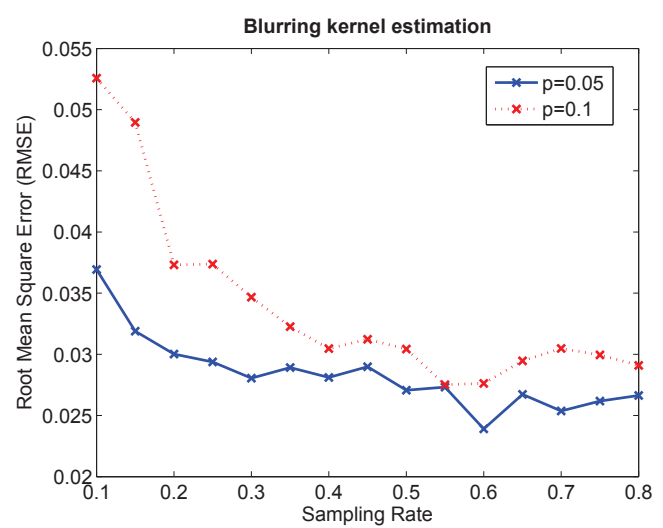

Fig. 5. Blurring kernel estimation error for various sampling rates for signal of sparsity equal to 0.02 (solid blue) and 0.05 (dashed red). We observe that higher sampling rates allow for a more accurate estimation of the kernel while the estimation process is more challenging at lower sparsity levels.

\section{CONCLUSIONS}

In this work we propose an extension of Compressed Sensing for sampling sparse signals that have undergone blurring with elements drawn from a blur dictionary. The reconstruction process is modeled as a series of alternating minimization problems between the spike signal estimation and the estimation of the sparse representation of the blurring kernel. Simulation results suggest that the proposed reconstruction scheme is able to achieve higher reconstruction performance compared to traditional CS based reconstruction schemes. Future work will include the investigation of different blurring dictionaries that may be able to encode a wider range of physical filtering processes.

\section{ACKNOWLEDGMENT}

This work was funded by the CS-ORION (PIAP-GA2009-251605) and HYDROBIONETS (ICT-GA-2011-287613) grants within the 7th Framework Program of the EU. 


\section{REFERENCES}

[1] Bruno A Olshausen, "Sparse codes and spikes," Probabilistic models of the brain - Perception and neural function, pp. 257-272, 2002.

[2] Ronen Tur, Yonina C Eldar, and Zvi Friedman, "Innovation rate sampling of pulse streams with application to ultrasound imaging," Signal Processing, IEEE Transactions on, vol. 59, no. 4, pp. 1827-1842, 2011.

[3] Matthew A Herman and Thomas Strohmer, "High-resolution radar via compressed sensing," Signal Processing, IEEE Transactions on, vol. 57, no. 6, pp. 2275-2284, 2009.

[4] Farid M Naini, Rémi Gribonval, Laurent Jacques, and Pierre Vandergheynst, "Compressive sampling of pulse trains - spread the spectrum," in Acoustics, Speech and Signal Processing, 2009. ICASSP 2009. IEEE International Conference on. IEEE, 2009, pp. 2877-2880.

[5] Zhilin Zhang, T Jung, Scott Makeig, and B Rao, "Compressed sensing of eeg for wireless telemonitoring with low energy consumption and inexpensive hardware," Biomedical Engineering, IEEE Transactions on, vol. 60, no. 1, pp. 221-224, 2013.

[6] D.L. Donoho, "Compressed sensing," Information Theory, IEEE Transactions on, vol. 52, no. 4, pp. 1289-1306, 2006

[7] Emmanuel J Candes, Yonina C Eldar, Deanna Needell, and Paige Randall, "Compressed sensing with coherent and redundant dictionaries," Applied and Computational Harmonic Analysis, vol. 31, no. 1, pp. 59$73,2011$.

[8] Emmanuel J Candès and Michael B Wakin "An introduction to compressive sampling," Signal Processing Magazine, IEEE, vol. 25, no. 2, pp. 21-30, 2008.

[9] Yaakov Tsaig and David L Donoho, "Extensions of compressed sensing," Signal processing, vol. 86, no. 3, pp. 549-571, 2006.

[10] Paul D O'Grady and Scott T Rickard, "Compressive sampling of nonnegative signals," in Machine Learning for Signal Processing, 2008. MLSP 2008. IEEE Workshop on. IEEE, 2008, pp. 133-138.

[11] Holger Rauhut, Karin Schnass, and Pierre Vandergheynst, "Compressed sensing and redundant dictionaries," Information Theory, IEEE Transactions on, vol. 54, no. 5, pp. 2210-2219, 2008

[12] S Derin Babacan, Rafael Molina, and Aggelos K Katsaggelos, "Variational bayesian blind deconvolution using a total variation prior," Image Processing, IEEE Transactions on, vol. 18, no. 1, pp. 12-26, 2009.

[13] Michael M Bronstein, Alexander M Bronstein, Michael Zibulevsky, and Yehoshua Y Zeevi, "Blind deconvolution of images using optimal sparse representations," IEEE Transactions on Image Processing, vol. 14, no. 6, pp. 726-736, 2005.

[14] Chinmay Hegde and Richard G Baraniuk, "Sampling and recovery of pulse streams," Signal Processing, IEEE Transactions on, vol. 59, no. 4, pp. 1505-1517, 2011.

[15] Tomer Michaeli and Yonina C Eldar, "Xampling at the rate of innovation," Signal Processing, IEEE Transactions on, vol. 60, no. 3 , pp. 1121-1133, 2012.

[16] Yonina C Eldar and Gitta Kutyniok, Compressed sensing - theory and applications, Cambridge University Press, 2012

[17] Anna Gilbert and Piotr Indyk, "Sparse recovery using sparse matrices," Proceedings of the IEEE, vol. 98, no. 6, pp. 937-947, 2010.

[18] Weizhi Lu, Weiyu Li, Kidiyo Kpalma, and Joseph Ronsin, "Near-optimal binary compressed sensing matrix," arXiv preprint arXiv:1304.4071, 2013.

[19] Robert M Gray, Toeplitz and circulant matrices: A review, Now Pub, 2006.

[20] Tony F Chan and Chiu-Kwong Wong, "Convergence of the alternating minimization algorithm for blind deconvolution," Linear Algebra and its Applications, vol. 316, no. 1, pp. 259-285, 2000.

[21] Filip Sroubek and Peyman Milanfar, "Robust multichannel blind deconvolution via fast alternating minimization," Image Processing, IEEE Transactions on, vol. 21, no. 4, pp. 1687-1700, 2012. 